\title{
Comparação da diversidade de comportamentos exibidos por emas em cativeiro e recém liberadas
}

\author{
Gilson de Souza Ferreira Neto ${ }^{1,2}$ (D), Marco Antônio Prado ${ }^{1,3}$, Pedro Ubatan \\ Camargo Neves $^{1,3}$ (D) Regison da Costa Oliveira ${ }^{2}$ (D) \& Elizabeth Ferreira \\ Guimarães $^{4}$
}

(1) Universidade Federal de Goiás - Campus II, Instituto de Ciências Biológicas, Avenida Esperança, Campus Samambaia 74690-900, Goiânia, Goiás, Brasil. E-mail: marcopradobio@gmail.com

(2) Instituto Nacional de Pesquisas da Amazônia, Departamento de Ecologia, Programa de PósGraduação em Biologia (Ecologia), Avenida André Araújo 2936, Petrópolis 69067-375, Manaus, Amazonas, Brasil. E-mail: gilsonsouzaferreiraneto@gmail.com

(3) Universidade Federal do Rio Grande do Sul, Instituto de Biociências, Departamento de Ecologia, Avenida Bento Gonçalves 9500, Agronomia 91501-970, Porto Alegre, Rio Grande do Sul, Brasil. Email: pedro.ubatan@gmail.com

(4) Associação Floresta Cheia Instituto de Conservação Ambiental, Rua b17, Residencial Recanto do Bosque, Goiânia, Goiás, Brasil. E-mail: organico.beth@gmail.com

Neto G.S.F., Prado M.A., Neves P.U.C., Oliveira R.C. \& Guimarães E.F. (2021) Comparação da diversidade de comportamentos exibidos por emas em cativeiro e recém liberadas. Pesquisa e Ensino em Ciências Exatas e da Natureza, 5: e1809. http://dx.doi.org/10.29215/pecen.v5i0.1809

Editor acadêmico: Silvio F. B. Lima. Recebido: 19 agosto 2021. Aceito: 01 outubro 2021. Publicado: 19 outubro 2021.

Resumo: Em geral, animais resgatados passam um período em cativeiro para avaliação da condição física e capacidade de sobrevivência antes de retornarem a natureza. Durante este período, os animais devem ser estimulados através de um enriquecimento ambiental e alimentar para manterem os comportamentos similares aos apresentados em meio natural. Este estudo foi realizado com seis indivíduos de Rhea americana no bioma Cerrado, em Goiás. O objetivo deste trabalho foi avaliar a diversidade de comportamentos de emas, através de uma comparação entre os comportamentos de cativeiro e vida livre. Os animais tinham entre 4 e 10 meses durante o monitoramento, sendo que os mesmos indivíduos foram observados em cativeiro e vida livre. Observações comportamentais foram feitas com seis indivíduos durante um total de 120 h, sendo 60 h em cativeiro e 60 h em vida livre. Para a construção do etograma foram utilizados os métodos de observação ad libitum e animal focal. Identificamos um total de 19 comportamentos agrupados em oito categorias comportamentais: alimentação, encontro agonístico, locomoção, limpeza, vocalização, brincadeiras, inatividade e defesa. As diversidades de comportamentos tiveram frequências semelhantes nas fases de cativeiro e vida livre. Os comportamentos de locomoção e vocalização tiveram maior expressão em cativeiro, enquanto os comportamentos de limpeza, alimentação, encontro agonístico e brincadeira tiveram maior expressão em vida livre. Entretanto, não houve nenhuma diferença significativa entre os comportamentos de vida livre e cativeiro. Nesse estudo foram feitas observações apenas de machos, e por isso, sugerimos estudos adicionais que incluam machos e fêmeas, para serem observados possíveis comportamentos de reprodução. As reintroduções com emas, geralmente, não são bem sucedidas, considerando a alta taxa de mortalidade por um predador natural. Medidas adequadas de manejo poderiam evitar estes problemas. Desta forma, ações conservacionistas que visem a reintrodução de emas e que identifiquem os comportamentos de indivíduos em cativeiro e vida livre podem ajudar a melhorar o bem-estar animal em cativeiro e, desta forma, aumentar as chances de sobrevivência no ambiente natural. $\mathrm{O}$ enriquecimento ambiental e alimentar pode ser essencial para estimular os comportamentos naturais, evitando comportamentos estereotipados em cativeiro. Além disso, os métodos usados nesse estudo poderiam ser utilizados para outras aves ratitas. 
Palavras chave: Aves ratitas, bem-estar animal, Cerrado, observações comportamentais, Rhea americana.

\title{
Comparison of the diversity of behaviors exhibited by captive and newly released rheas
}

\begin{abstract}
In general, rescued animals spend a period in captivity to assess their physical condition and survivability before returning to the wild. During this period, animals must be stimulated through environmental and food enrichment to maintain behaviours similar to those presented in the natural environment. This study was carried out with six individuals from Rhea americana in the Cerrado biome in Goiás. The goal of this study was to evaluate the diversity of greater rhea behaviours through a comparison between animal behaviours in captivity and the wild. The animals had between 4 and 10 months during monitoring, and the same individuals were observed in captivity and the wild. Behavioural observations were made with six individuals over a total of 120 hours, with 60 hours in captivity and 60 hours in the wild. To compile the ethogram, ad libitum and focal animal observation methods were used. We identified a total of 19 behaviours grouped into eight behavioural categories: feeding, agonistic interaction, locomotion, self-maintenance, vocalization, playing, inactivity and self-defence. The diversity of behaviours had similar rates in captivity and the wild. The behaviours of locomotion and vocalization had greater expression in captivity, while the behaviours of self-maintenance, feeding, agonistic interaction, and playing had greater expression in the wild. However, there was no significant difference between animal behaviours in the wild and captivity. In this study, observations were made only from males, and for this reason, we suggest additional studies including males and females, to observe possible reproductive behaviours. Reintroductions with greater rhea are generally unsuccessful, considering that a natural predator kills them. Adequate management measures could avoid these problems. In this way, conservationist actions can help to improve animal welfare in captivity and, therefore, increase the chances of survival in the natural environment. Environmental and food enrichment can be essential to stimulate natural behaviours, avoiding stereotyped behaviours in captivity. In addition, the methods used in this study could be used for other ratite birds.
\end{abstract}

Key words: Animal welfare, behavioural observations, Cerrado, ratite birds, Rhea Americana.

\section{Introdução}

Uma forma de aumentar o conhecimento das espécies é através de estudos comportamentais, tanto em cativeiro como em vida livre (Marini \& Marinho-Filho 2006; Neto et al. 2020, 2021). Os estudos comportamentais têm tido um papel importante na conservação de espécies (Webb et al. 2020). A partir da avaliação do estado comportamental de animais, tem sido possível auxiliar pesquisadores na construção de ambientes em cativeiro com melhor qualidade (Caro 2007) e avaliar o sucesso de estratégias de reintrodução ou reforço populacional (Marini \& Marinho-Filho 2006). Por outro lado, de modo geral, existe uma lacuna de conhecimento das espécies nos trópicos sobre tais questões e, por isso, uma necessidade de se conhecer mais profundamente o comportamento das espécies.

A ema, Rhea americana, é a maior ave ratita das américas, com altura variando entre 134 e $170 \mathrm{~cm}$ (Gwynne et al. 2010). A espécie é nativa da região neotropical ocorrendo na Argentina, Bolívia, Paraguai, Uruguai e vários estados do Brasil, preferencialmente, em ambientes de pastagem e savana como o Cerrado (Del Hoyo et al. 1992). A espécie é onívora e a alimentação consiste de folhas, flores, frutos, sementes, invertebrados e pequenos vertebrados, como lagartixas e roedores (Azevedo \& Young 2006; Giordano et al. 2010; Comparatore \& Yagueddú 2016). As maiores populações naturais encontram-se nos estados do Mato Grosso e Goiás (Gunski 1992). Durante a época de reprodução, que se estende de agosto a fevereiro, nas pradarias do Rio Grande do Sul (Codenotti 1997), os grupos se separam em pequenos bandos e os machos se tornam bastante agressivos (Carro \& Fernández 2008). O macho adulto é mais robusto, possui o pescoço mais grosso com a base negra, peito e parte mediana do dorso anterior também negros, conferindo um leve dimorfismo sexual (Hone \& Mallon 2017). A ema tem um papel ecossistêmico importante como dispersora de sementes e como presa de várias espécies selvagens, como onça pintada (Panthera onca), lobo-guará (Chrysocyon brachyurus), cachorrovinagre (Speothos venaticus) e teiú-branco (Tupinambis teguixin) (Dani 1993; Azevedo \& Young 2006; Azevedo 2010). Desta forma, a possível extinção das emas em sua área de ocorrência pode 
diminuir as taxas de dispersão de sementes e a regeneração do ecossistema (Magnani \& Pascoal 1990; Renison et al. 2010).

Segundo a IUCN (2016), a ema é classificada globalmente como quase ameaçada devido aos aparentes declínios populacionais observados nos últimos anos, sendo que a população tem diminuído drasticamente nos estados brasileiros. A caça intensiva tem contribuído para a diminuição das populações desta ave em seu habitat natural, além de outras ações antrópicas como queimadas, perda de habitat para monoculturas em biomas como o Cerrado bem como a eliminação de ovos por maquinário agrícola (Dani 1993; Giannoni 1996). Além disso, muitos programas de reintrodução ou reforço populacional dessa espécie tem falhado (Beck et al. 1994; Wolf et al. 1996) e um dos motivos para isso tem sido a predação natural, uma vez que os animais são soltos na natureza de forma inadequada (Short et al. 1992; Miller et al. 1994). Devido ao declínio populacional desta ave ratita em várias escalas regionais, tornam-se necessárias iniciativas para identificar e caracterizar os comportamentos mais frequentes, tendo em vista melhorar as perspectivas de conservação e de solturas bem sucedidas (Erize \& Villafañe 2016). Nesse contexto, o presente estudo teve como objetivo identificar os comportamentos mais comuns em cativeiro e vida livre, além de comparar a exibição de comportamentos de $R$. americana observados durante o cativeiro e a vida livre.

\section{Material e Métodos}

\section{Área de estudo}

O estudo foi conduzido na fazenda Vale do Tamanduá (1659'25.2" S, 49²4'17.2" W), localizada no município de Aragoiânia, Goiás (Figura 1). A fazenda é uma área de soltura de animais silvestres com 99 hectares e está cadastrada no Instituto Brasileiro do Meio Ambiente (IBAMA). O local conta com a assistência de uma bióloga que acompanha diariamente os animais reintroduzidos. A área da fazenda inclui vegetação nativa e diferentes fisionomias de Cerrado, como Cerradão e campo cerrado, além de florestas de galeria (Eiten 1978).

\section{Coleta de comportamentos}

Seis animais foram utilizados na realização do presente estudo, sendo todos machos, visto que as fêmeas morreram antes do início do monitoramento. Os mesmos indivíduos que foram observados em cativeiro também foram observados em vida livre. As aves foram marcadas para observação, inicialmente com braçadeiras de náilon coloridas nas pernas, e não houveram tentativas de arrancamento. Na fase de vida livre, os indivíduos foram marcados com tinta de impressora com duas cores diferentes, em distintas partes do corpo, facilitando a identificação.

Os animais tiveram seu contato com humanos reduzidos ao máximo para evitar possíveis conflitos (e.g., comportamentos agressivos) após a soltura (Sekar et al. 2008). Os indivíduos foram alocadas em recintos com aspectos semelhantes ao seu habitat. $\mathrm{O}$ recinto destinado aos indivíduos reintroduzidos tinha cerca de seis metros de comprimento, catorze metros de largura e dois metros de altura, bebedouro com água reposta duas vezes ao dia, comedouro e vegetação constituída por gramíneas que eram usadas pelos animais na alimentação. A alimentação era fornecida duas vezes ao dia, uma pela manhã, por volta das $07 \mathrm{~h}$; e uma durante a tarde, aproximadamente às $16 \mathrm{~h}$. $\mathrm{O}$ recinto era limpo semanalmente e oferecido um enriquecimento alimentar e ambiental. A dieta foi desenvolvida por veterinários do Centros de Triagem de Animais Silvestres (Cetas) do Instituto Brasileiro do Meio Ambiente e dos Recursos Naturais Renováveis (IBAMA), e era constituída por uma ração balanceada composta de $1 \mathrm{~kg}$ de folhagens diversas como couve, almeirão, folhas de cenoura, beldroega, frutas nativas da região e insetos (tenébrios) espalhadas no recinto para diminuir possíveis conflitos entre os indivíduos. 


\section{Comportamento de Rhea americana}

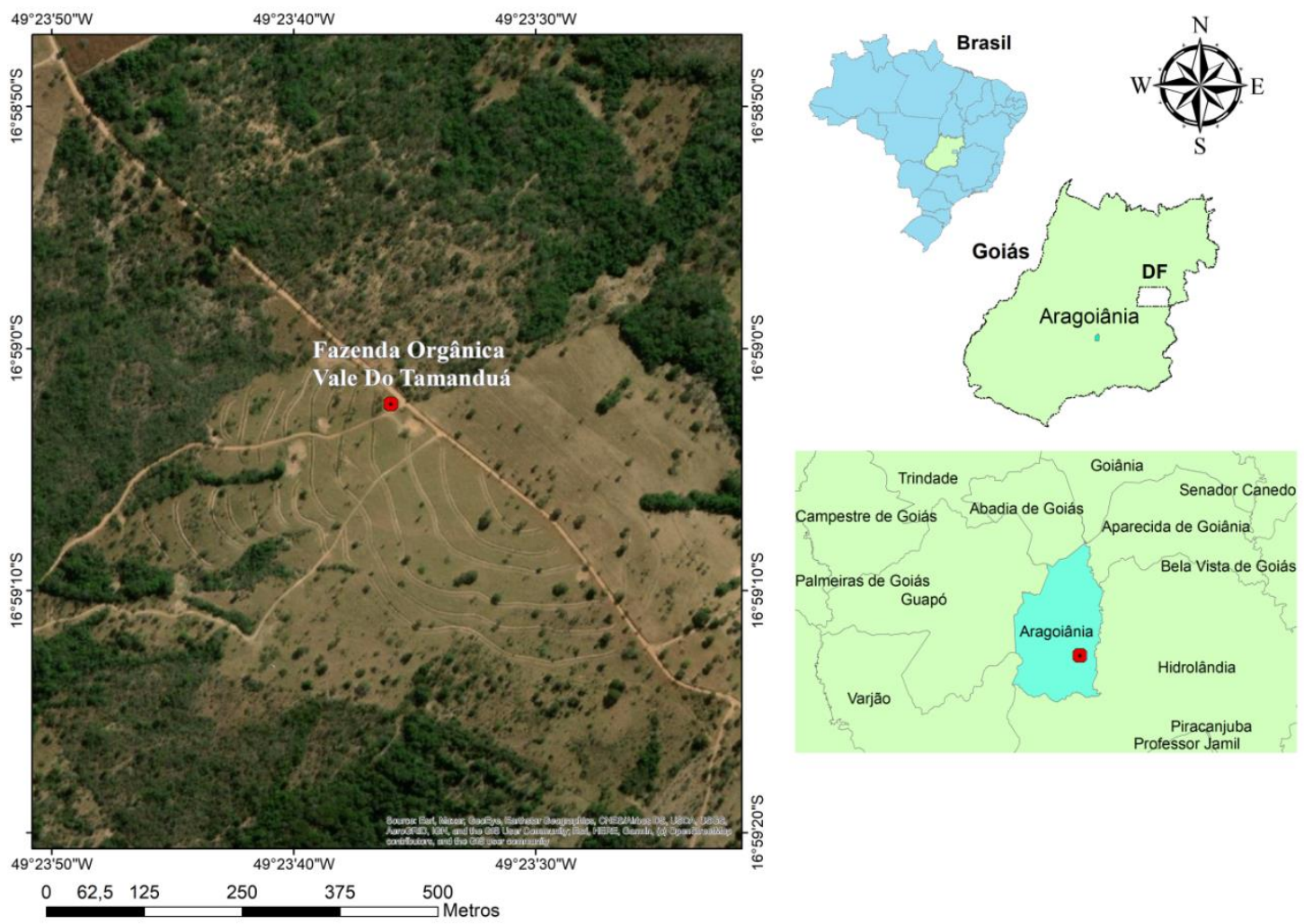

Figura 1. Mapa da área de estudo no município de Aragoiânia, Goiás.

O estudo foi realizado entre novembro de 2013 e abril de 2014 , aos finais de semana entre às 07 e $20 \mathrm{~h}$, sendo que $72.87 \%$ das observações foram feitas nos horários de 09 às $16 \mathrm{~h}$, visto que o período de maior atividade das emas ocorre no início do período matutino (6:00 às 10:00 am) e o início do período vespertino (12:00 às 16:00 pm) em diferentes tipos de habitats (Azevedo et al. 2010). O estudo contou com um total de $120 \mathrm{~h}$ de observação, sendo $60 \mathrm{~h}$ em cativeiro e $60 \mathrm{~h}$ em vida livre. Para as análises, considerou-se apenas os períodos matutino e vespertino. A princípio, a técnica amostragem de todas as ocorrências (ad libitum) foi utilizada para a realização do etograma, a fim de identificar todos os comportamentos dos animais (Del-Claro et al. 2004). Após a padronização dos comportamentos, cada animal foi observado por 10 minutos e todos os comportamentos neste tempo foram registrados. A coleta de dados foi dividida nas fases de cativeiro e vida livre para compor o etograma. Todos os indivíduos chegaram recém-nascidos na fazenda, mas durante o monitoramento eram jovens entre 4 e 10 meses. Neste estudo foi possível seguir e visualizar facilmente os animais devido a área de monitoramento ser um Cerrado aberto (Figura 2).

\section{Análise de dados}

Para comparar os comportamentos em cativeiro e vida livre, foi calculado o índice de diversidade de comportamentos (IDC) entre os ambientes de vida livre e cativeiro. O IDC considera o número e a proporção dos comportamentos exibidos; onde valores mais altos equivalem a melhores níveis de bem-estar (Shepherdson et al. 1993). O cálculo do IDC (= H) é realizado através da fórmula: $H=\Sigma$ pi.log (1/pi) (1), onde pi é a abundância relativa de cada categoria comportamental, calculada como a proporção de elementos comportamentais de uma categoria pelo número total de comportamentos (Shepherdson et al. 1993). O teste não paramétrico de Wilcoxon também foi usado para avaliar a significância dos comportamentos entre cativeiro e vida livre (Wilcoxon 1992). 


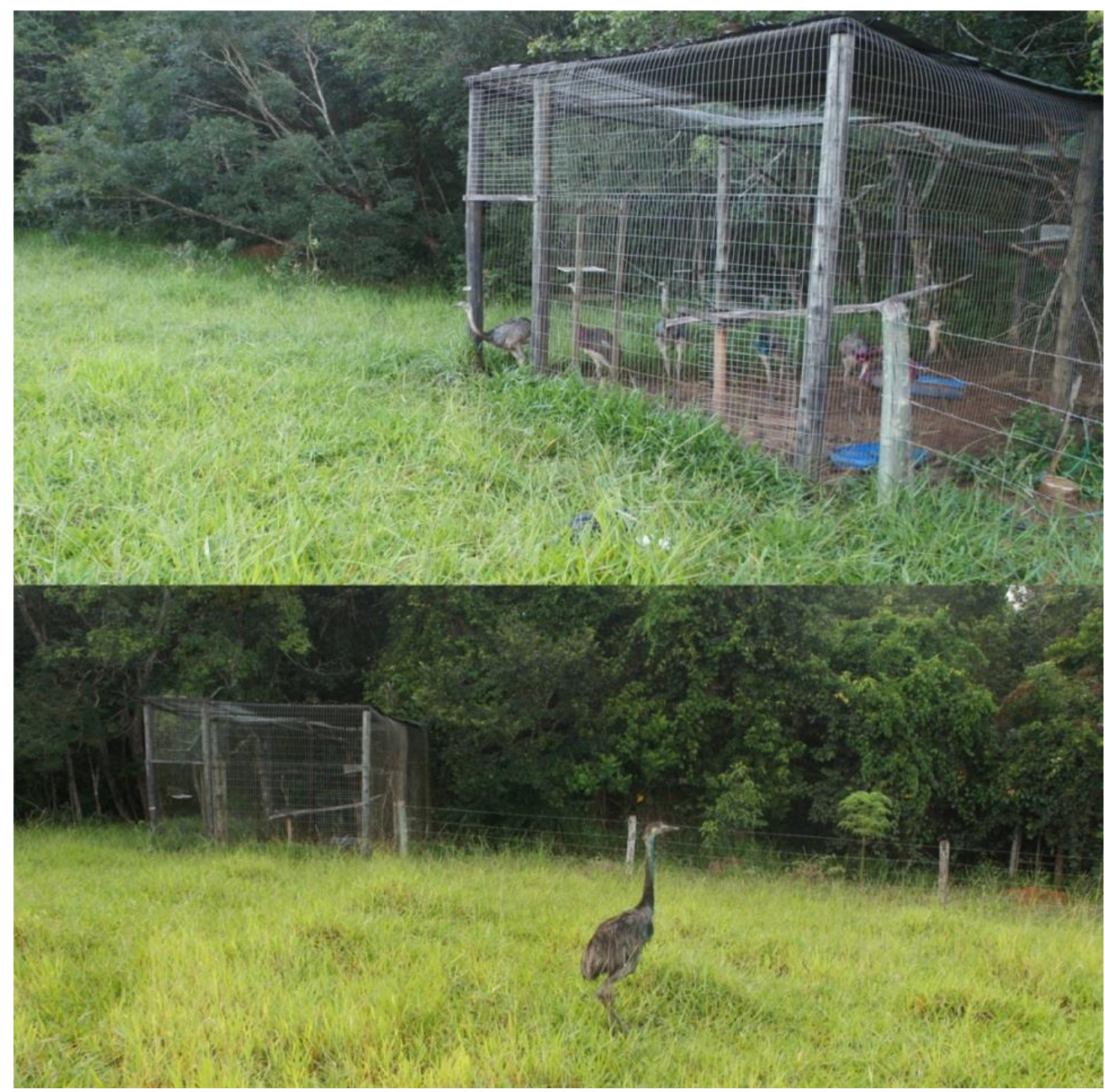

Figura 2. Ambiente de Cerrado aberto onde o grupo de emas (R. americana) foi monitorado.

\section{Resultados}

Um etograma foi feito para emas (Tabela 1), e baseado na literatura (Codenotti et al. 1995; Azevedo et al. 2012). Foram identificados 19 tipos diferentes de comportamentos agrupados em oito categorias comportamentais: locomoção, limpeza, alimentação, encontro agonístico, vocalização, brincadeira, inatividade e defesa em cativeiro e vida livre (Figura 2). Os comportamentos mais comuns foram forragear $(36.36 \%)$, comer $(22.27 \%)$ e pentear as penas (9.32\%), enquanto os comportamentos menos comuns foram encontro agonístico (0.99\%), coçar $(0.64 \%)$, brincadeira $(0.21 \%)$, espreguiçar $(0.35 \%)$, bocejar $(0.16 \%)$, vocalizar $(0.13 \%)$ e pular para capturar alimento $(0.05 \%)$.

As categoria comportamentais mais frequentes neste estudo foram locomoção, limpeza e alimentação, enquanto os comportamentos menos exibidos foram vocalização, brincadeira, encontro agonístico, inatividade e defesa para cativeiro e vida livre. Ao todo, foram 1953 registros comportamentais em cativeiro e 1779 em vida livre (Figura 3).

Em geral, a quantidade de comportamentos em vida livre e cativeiro foram semelhantes, e não houve diferença significativa para locomoção ( $w=16.50 ; \mathrm{p}=0.87$ ), limpeza $(\mathrm{w}=15 ; \mathrm{p}=$ $0.68)$, alimentação ( $w=15.50 ; p=0.73)$, encontro agonístico $(w=29 ; p=0.06)$, vocalização $(w=9$; $\mathrm{p}=0.11)$, brincadeira $(\mathrm{w}=26 ; \mathrm{p}=0.18)$, defesa $(\mathrm{w}=30 ; \mathrm{p}=0.063)$ e inatividade $(\mathrm{w}=15.5 ; \mathrm{p}=$ 0.74). Proporcionalmente ao número total de comportamentos em cativeiro, os comportamentos 


\section{Comportamento de Rhea americana}

de locomoção (IDC $=0.1597)$, limpeza $($ IDC $=0.13)$, alimentação (IDC $=0.15)$, vocalização $($ IDC $=$ 0.005) e defesa (IDC $=0.05$ ) tiveram maior IDC em relação a vida livre, enquanto os comportamentos encontro agonístico (IDC $=0.034)$, brincadeira (IDC $=0.008$ ) e inatividade (IDC $=0.114$ ) foram maiores proporcionalmente em vida livre (Tabela 2).

Tabela 1. Descrição dos comportamentos observados e categorias correspondentes a seis indivíduos de $R$. americana em cativeiro.

\begin{tabular}{|c|c|c|c|c|c|}
\hline Categoria comportamental & Atividade & Descrição & Cativeiro & Vida livre & $\%$ \\
\hline \multirow{4}{*}{ Alimentação } & Beber & A ave ingere água de pé ou deitada. & 91 & 10 & 2.71 \\
\hline & Comer & $\begin{array}{l}\text { O indivíduo de pé ou deitado bica e } \\
\text { ingere o seu alimento. }\end{array}$ & 422 & 409 & 22.27 \\
\hline & Coprofagia & $\begin{array}{l}\text { O animal de pé ou sentado bica e ingere } \\
\text { fezes. }\end{array}$ & 114 & 09 & 3.30 \\
\hline & $\begin{array}{l}\text { Pular para } \\
\text { capturar } \\
\text { alimento }\end{array}$ & $\begin{array}{l}\text { Animal eleva-se do chão por impulso } \\
\text { dos pés e das pernas para capturar o } \\
\text { alimento. }\end{array}$ & 01 & 01 & 0.05 \\
\hline Brincadeira & Brincadeira & $\begin{array}{l}\text { Os indivíduos correm em linha reta ou } \\
\text { em curso irregular. Algumas vezes este } \\
\text { comportamento era seguido ou iniciado } \\
\text { com saltos, sendo que após um indivíduo } \\
\text { começar a atividade, as outras aves } \\
\text { repetem. }\end{array}$ & 02 & 06 & 0.21 \\
\hline \multirow{2}{*}{ Locomoção } & Forragear & $\begin{array}{l}\text { Quando o animal se locomove de forma } \\
\text { lenta, seja para somente deslocar-se ou } \\
\text { para procurar alimento. }\end{array}$ & 666 & 691 & 36.36 \\
\hline & Correr & $\begin{array}{l}\text { Ocorre quando o animal se locomove de } \\
\text { forma rápida em um curto espaço de } \\
\text { tempo. }\end{array}$ & 24 & 30 & 1.45 \\
\hline \multirow{7}{*}{ Limpeza } & Bocejar & $\begin{array}{l}\text { Animal abre o bico, mantendo-o aberto } \\
\text { por algum tempo, ao mesmo tempo que } \\
\text { mantém os olhos fechados. }\end{array}$ & 03 & 03 & 0.16 \\
\hline & Coçar & $\begin{array}{l}\text { A ave esfrega alguma parte do corpo } \\
\text { utilizando suas patas. }\end{array}$ & 11 & 13 & 0.64 \\
\hline & Defecar & O indivíduo expele fezes. & 33 & 21 & 1.45 \\
\hline & Espreguiçar & $\begin{array}{l}\mathrm{O} \text { animal permanece parado, esticando } \\
\text { uma das pernas, estendendo o pescoço } \\
\text { para frente e levanta a cabeça. }\end{array}$ & 11 & 02 & 0.35 \\
\hline & $\begin{array}{l}\text { Sacundindo as } \\
\text { penas }\end{array}$ & $\begin{array}{l}\mathrm{O} \text { animal eriça e balança as penas, } \\
\text { levantando as asas. }\end{array}$ & 98 & 54 & 4.07 \\
\hline & $\begin{array}{l}\text { Pentear as } \\
\text { penas }\end{array}$ & $\begin{array}{l}\text { O indivíduo de pé ou deitado inclina sua } \\
\text { cabeça em direção às asas usando o bico } \\
\text { para alisar e limpar suas asas. }\end{array}$ & 157 & 191 & 9.32 \\
\hline & $\begin{array}{l}\text { Tomar banho } \\
\text { de terra }\end{array}$ & $\begin{array}{l}\text { Em posição sentada o indivíduo leva seu } \\
\text { pescoço para trás pegando terra do chão } \\
\text { com o bico e jogando-a em seu dorso. }\end{array}$ & 32 & 29 & 1.63 \\
\hline Encontro Agonístico & $\begin{array}{l}\text { Encontro } \\
\text { Agonístico }\end{array}$ & $\begin{array}{l}\text { A ave corre ao encontro de outro } \\
\text { indivíduo, bicando o seu corpo. Por } \\
\text { vezes, antes da agressão a ave ameaçava } \\
\text { o oponente rondando o outro com o bico } \\
\text { aberto até começar o ataque em si. Este } \\
\text { comportamento geralmente ocorria } \\
\text { durante o período de alimentação. }\end{array}$ & 01 & 36 & 0.99 \\
\hline Vocalização & Vocalizar & A ave produz um som. & 04 & 01 & 0.13 \\
\hline \multirow[b]{2}{*}{ Inatividade } & Parado & $\begin{array}{l}\text { A ave permanece em pé, parada sem } \\
\text { executar nenhum outro comportamento. }\end{array}$ & 46 & 52 & 2.63 \\
\hline & $\begin{array}{l}\text { Descansar/ } \\
\text { Dormir }\end{array}$ & $\begin{array}{l}\text { A ave sentada, inclina seu pescoço para } \\
\text { frente, repousando a cabeça entre as } \\
\text { asas, ou estira o pescoço sobre o chão } \\
\text { com a cabeça voltada para frente. }\end{array}$ & 165 & 176 & 9.14 \\
\hline Defesa & Vigília & $\begin{array}{l}\text { O animal fica em posição de alerta } \\
\text { erguendo o pescoço e fixando o olhar } \\
\text { em algum local durante algum tempo. }\end{array}$ & 72 & 45 & 3.14 \\
\hline
\end{tabular}




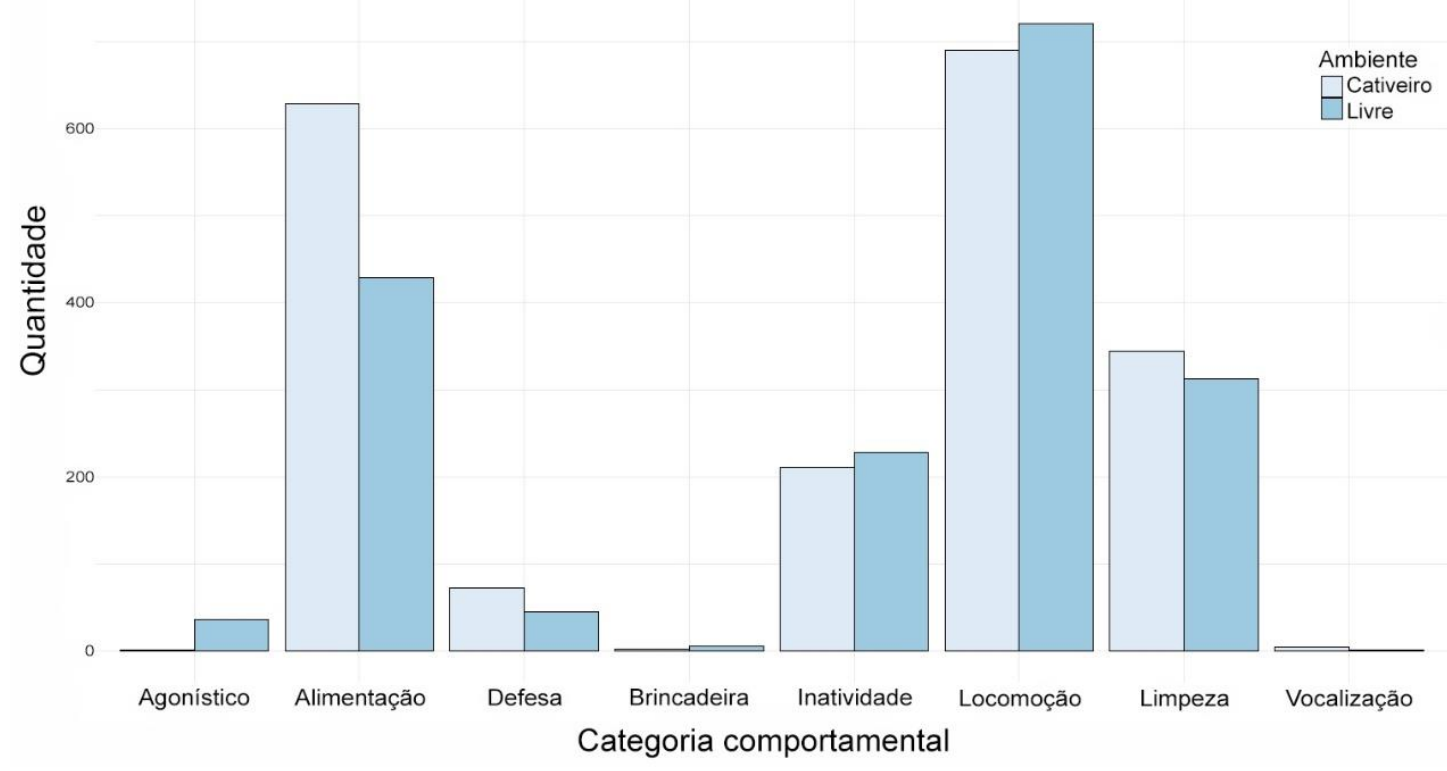

Figura 3. Número total de comportamentos em cada categoria comportamental em dois ambientes diferentes: cativeiro e vida livre.

Tabela 2. Resumo dos resultados do teste não paramétrico Wilcoxon, e do índice de diversidade de comportamentos, mostrando a variação de comportamentos entre cativeiro e vida livre $(\mathrm{N}=6)$.

\begin{tabular}{llllllc}
\hline Comportamentos & Ambiente & IDC & $\%$ & Qtd & W & P \\
\hline Locomoção & Cativeiro & 0.159 & 36.680 & 690 & 16.500 & 0.870 \\
& Vida livre & 0.158 & 40.520 & 721 & & 0.688 \\
\hline Limpeza & Cativeiro & 0.135 & 18.340 & 345 & 15 & \\
& Vida livre & 0.132 & 17.590 & 313 & & 0.732 \\
\hline Alimentação & Cativeiro & 0.159 & 33.380 & 628 & 15.500 & \\
\hline Encontro agonístico & Vida livre & 0.148 & 24.110 & 429 & & 0.060 \\
& Cativeiro & 0.001 & 0.050 & 01 & 29 & 0.112 \\
\hline Vocalização & Vida livre & 0.034 & 2.020 & 36 & & 0.189 \\
& Cativeiro & 0.005 & 0.210 & 04 & 09 & \\
\hline Brincadeira & Vida livre & 0.001 & 0.050 & 01 & & 0.063 \\
& Cativeiro & 0.003 & 0.100 & 02 & 26 & \\
\hline Defesa & Vida livre & 0.008 & 0.330 & 06 & & 0.748 \\
\hline Inatividade & Cativeiro & 0.054 & 3.820 & 72 & 30 & \\
& Vida livre & 0.040 & 2.520 & 45 & & 15.500 \\
\hline
\end{tabular}

\section{Discussão}

Estudos etológicos de $R$. americana estão disponíveis na literatura (Codenotti et al. 1995; Azevedo et al. 2010; Azevedo et al. 2012), e dados sobre deslocamento de emas na natureza (Bellis et al. 2004). A categoria comportamental locomoção foi a com maior porcentagem (36.68\% para cativeiro e $40.52 \%$ em vida livre) do total dos comportamentos observados. Entre os comportamentos inseridos na categoria locomoção, forrageamento foi o comportamento mais frequente para a espécie em cativeiro e vida livre, corroborando com dados de outros trabalhos para a fase de vida livre em emas (Azevedo et al. 2010). Locomoção é um comportamento chave para vários outros tipos de comportamentos como encontro agonístico, alimentação e forrageamento, e que aumenta a sobrevivência do indivíduo (Lewis \& Hurnik 1990). 
Comportamentos estereotipados foram observados nesse estudo, mas em proporção insignificante. Estudos etológicos indicam que comportamentos estereotipados como se locomover de um lado para outro sem motivo aparente pode ser patológico e um indicativo de estresse devido ao espaço reduzido (Ödberg 1984) ou um problema de bem-estar (Fraser \& Broom 1997). No entanto, cabe ressaltar que o que pode definir um comportamento estereotipado se for patológico ou não é o contexto ambiental que o animal está inserido (Mason 1991).

Neste estudo também foi observado o comportamento brincadeira $(0.10 \%$ em cativeiro e $0.33 \%$ em vida livre), assim como foi observado em estudos anteriores (Azevedo et al. 2012). Os indivíduos correm, e depois este comportamento é seguido ou iniciado com saltos, sendo que após um indivíduo começar a atividade, a outra ave tende a repetir. As ocorrências observadas podem ser confundidas com fuga ou como correr sem motivo aparente, mas diferente de fuga, as aves não demonstram outras características como cabeça erguida acima do tronco que indicaria um porte defensivo (Ficken 1977). Correr sem a utilização das asas também é um comportamento comun (Codenotti et al. 1995). Este comportamento é geralmente descrito para aves precociais (Azevedo et al. 2012). Estas observações corroboram com as ocorrências em $R$. americana. McBride et al. (1969), estudando galináceos selvagens, observou um comportamento de brincadeira de luta que era iniciado com os animais correndo em linha reta ou em cursos irregulares, e em seguida assumiram uma posição de confronto. Estas ocorrências se assemelham muito com o que foi observado em $R$. americana, exceto no término da atividade que no caso das emas não havia uma posição de luta.

A categoria encontro agonístico teve maior frequência em vida livre $(0.05 \%$ em cativeiro e $2.02 \%$ em vida livre). Esse resultado provavelmente pode ser justificado porque em vida livre os indivíduos tinham uma idade mais avançada, o que sinaliza o início da maturação sexual e puberdade (Martella et al. 1994; Codenotti \& Alvarez 2001). Em cativeiro registrou-se apenas uma ocorrência, enquanto em vida livre foram 36 registros desse comportamento. Os animais ficaram sempre próximos e não se dispersaram. Os comportamentos de limpeza também estiveram presentes tanto em cativeiro (18.34\%) como em vida livre (17.59\%). O aumento de comportamentos de limpeza em cativeiro como defecar, espreguiçar, sacudir as penas e tomar banho de terra, assim como de comportamentos de defesa como vigília, podem ser um indicativo de estresse comportamental fisiológico ou medo dependendo do contexto de exibição , sendo que o gatilho para tais comportamentos podem ser a presença de um predador ou brigas (Munksgaard et al. 1997; Moberg \& Mench 2000; Azevedo \& Young 2006). As emas aumentam a vigilância quando se sentem ameaçadas por possíveis predadores como onça pintada (Panthera onca), puma (Puma concolor), lobo-guará (Chrysocyon brachyurus) e cachorro-vinagre (Speothos venaticus) (Dani 1993), ou na presença de humanos (Azevedo \& Young 2006; Azevedo et al. 2012). Além disso, emas machos exibem com mais frequência esses comportamentos de defesa do que as fêmeas (Reboreda \& Fernandez 1997).

O presente estudo traz a descrição dos comportamentos de emas, e compara a diversidade de comportamentos entre cativeiro e vida livre. Além disso, os métodos usados nesse estudo podem ser utilizados para outras aves ratitas. Os comportamentos expressos pelas emas neste estudo podem ter sido afetados por outros fatores que não foram quantificados como a diferença de idade entre os mesmos indivíduos de vida livre e cativeiro. Além disso, as emas apresentaram alguns comportamentos redundantes, especialmente em cativeiro, o que pode ter comprometido o bem estar dos indivíduos deste estudo. Encorajamos novos estudos que avaliem outros fatores que podem afetar o comportamento das emas, como por exemplo, diferenças de comportamentos entre machos e fêmeas. Além disso, programas que considerem um treinamento anti-predador para que as emas consigam reconhecer os predadores naturais, e iniciativas de educação da população onde os indivíduos serão soltos, para aumentar as chances de reintrodução ou reforço populacional bem sucedido e com menores possibilidades de predação em um ambiente natural. Outrossim, auxiliar pesquisadores na construção de cativeiros com melhor qualidade e enriquecimento ambiental e alimentar. Tais informações são 
importantes e necessárias para a conservação de tal espécie quase ameaçada, permitindo melhores decisões em planos de manejo, tanto no ambiente natural, como em cativeiro.

\section{Agradecimentos}

Somos gratos principalmente aos proprietários da área onde os estudos foram realizados, e ao Centro de Triagem de Animais Silvestres de Goiás (CETAS / IBAMA-GO), pelo apoio logístico e material.

\section{Referências}

Azevedo C.S. (2010) Ecologia, comportamento e manejo de emas (Rhea americana, Rheidae, Aves). Tese (Programa de Pós-Graduação em Ecologia, Conservação e Manejo de Vida Silvestre). Universidade Federal de Minas Gerais, Instituto de Ciências Biológicas, Belo Horizonte, Minas Gerais.

Azevedo C.S. \& Young R.J. (2006) Behavioral responses of captive-born greater rheas (Rhea americana) Linnaeus (Rheiformes, Rheidae) submitted to antipredator training. Revista Brasileira de Zoologia, 23: 186-193. https://doi.org/10.1590/S0101-81752006000100010

Azevedo C.S., Ferraz J.B., Tinoco H.P., Young R.J. \& Rodrigues M. (2010) Time-activity budget of greater rheas (Rhea americana, Aves) on a human-disturbed area: the role of habitat, time of the day, season and group size. Acta ethologica, 13: 109-117. https://doi.org/10.1007/s10211-010-0080-7

Azevedo C.S.D., Lima M.F.F., Silva V.C.A.D., Young R.J. \& Rodrigues M. (2012) Visitor influence on the behavior of captive greater rheas (Rhea americana, Rheidae Aves). Journal of Applied Animal Welfare Science, 15: 113-125. https://doi.org/10.1080/10888705.2012.624895

Beck B.B., Rapaport L.G., Stanley Price M.R. \& Wilson A.C. (1994) Reintroduction of captive-born animals (p. 265-286). In: Olney P.J.S., Mace G.M. \& Feistner A.T.C. (Eds). Creative Conservation: Interactive Management of Wild and Captive Animals. London: Chapman and Hall. 501 p.

Bellis L.M., Martella M.B., Navarro J.L. \& Vignolo P.E. (2004) Home range of greater and lesser rhea in Argentina: relevance to conservation. Biodiversity \& Conservation, 13: 2589-2598. https://doi.org/10.1007/s10531-004-1086-0

Caro T. (2007) Behavior and conservation: a bridge too far? Trends in Ecology and Evolution, 22: 394-400. https://doi.org/10.1016/j.tree.2007.06.003

Carro M.E. \& Fernández G.J. (2008) Seasonal variation in social organisation and diurnal activity budget of the Greater Rhea (Rhea americana) in the Argentinean Pampas. Emu-Austral Ornithology, 108: 167-173.

Codenotti T.L. (1997) Fenología reproductiva y biometría definidos, huevos y pollos del ñandú, Rhea americana en Rio Grande do Sul, Brasil. El Hornero, 14: 211-223.

Codenotti T.L. \& Alvarez F. (2001) Mating behavior of the male Greater Rhea. The Wilson Journal of Ornithology, 113: 85-89.

Codenotti T.L.D., Beninca \& F. Alvarez (1995) Etograma y relacion de la conducta con el habitat y con la edad en el ñandú. Doñana, Acta Vertebrata, 22: 65-86.

Comparatore V. \& Yagueddú C. (2016) Diet preference and density of the Greater Rhea (Rhea americana) in grasslands of the Flooding Pampa, Argentina. Ornithology Research, 24: 1320.

Dani S. (1993) A Ema Rhea americana: Biologia, manejo e conservação. Belo Horizonte: Q21 Fundação Acangaú. 136 p.

Del-Claro K., Prezoto F. \& Sabino J. (2004) Comportamento animal. Uma introdução à Ecologia Comportamental (p. 11-15). Jundiaí: Livraria Conceito. 134 p.

Del Hoyo J., Elliot A. \& Sargatal J.A. (1992) Handbook of the Birds of the World. Barcelona: Lynx Editions. 696 p.

Eiten G. (1978) Delimitation of the cerrado concept. Vegetatio, 36: 169-178. 
https://doi.org/10.1007/BF02342599

Erize F.G. \& Villafañe I.G. (2016) Habitats selected by the endangered Greater Rhea (Rhea americana) - implications for conservation. Emu-Austral Ornithology, 116: 379-386. https://doi.org/10.1071/MU15124

Ficken M.S. (1977) Avian play. The Auk, 94: 573-582.

Fraser A.F. \& Broom D.M. (1997) Farm animal behaviour and welfare. $3^{\circ}$ edição. Wallingford, England: CAB International. $437 \mathrm{p}$.

Giannoni M.L. (1996) Emus and ostriches: an alternative for the farmer. Jaboticabal: FUNEP. 49 p.

Giordano P.F., Navarro J.L. \& Martella M.B. (2010) Building large-scale spatially explicit models to predict the distribution of suitable habitat patches for the Greater Rhea (Rhea americana), a near-threatened species. Biological Conservation, 143: 357-365. https://doi.org/10.1016/j.biocon.2009.10.022

Gunski R.J. (1992) Análise citogenética e algumas considerações biológicas da espécie Rhea americana-Ema (Aves: Rheidae). Jaboticabal: Unesp. 129 p.

Gwynne J.A., Ridgely R.S., Argel M. \& Tudor G. (2010) Guia Aves do Brasil: Pantanal e Cerrado. São Paulo: Editora Horizonte. 322 p.

Hone D.W. \& Mallon J.C. (2017) Protracted growth impedes the detection of sexual dimorphism in non-avian dinosaurs. Palaeontology, 60: 535-545. https://doi.org/10.1111/pala.12298

IUCN (2016) BirdLife International: Rhea americana. The IUCN Red List of Threatened Species. Disponível em: https://dx.doi.org/10.2305/IUCN.UK.2013.RLTS.T22678073A92754472.en (Acessado em: 13/08/2021).

Lewis N.J. \& Hurnik J.F. (1990) Locomotion of broiler chickens in floor pens. Poultry Science, 69: 1087-1093. https://doi.org/10.3382/ps.0691087

Magnani F.S. \& Paschoal F.R. (1990) Dispersão de sementes pela ema (Rhea americana) em condições de cativeiro (p. 172). Resumos: XVII Congresso Brasileiro de Zoologia. Londrina: SBZ.

Marini M.A. \& Marinho-Filho J.S. (2006) Translocação de aves e mamíferos: teoria e prática no Brasil (p. 505-533). In: Rocha C.F.D., Bergallo H.G., Van Sluys M. \& Alves M.A.S. Biologia da Conservação. São Carlos: RIMA. 582 p.

Martella M.B., Navarro J.L., Sahade R., Tatián M. \& Burgos A. (1994) Breeding system of the Greater Rhea, Rhea americana. Journal of Ornithology, 135: 123.

Mason G.J. (1991) Stereotypies and suffering. Behavioural Processes, 25: 103-115. https://doi.org/10.1016/0376-6357(91)90013-P

McBride G.I., Farer P. \& Foenander F. (1969) The social organization and behavior of the feral domestic fowl. Animal Behaviour Monographs, 2: 127-181. https://doi.org/10.1016/S0066-1856(69)80003-8

Miller B., Biggins D., Hanebury L., Vargas A. (1994) Reintroduction of the black-footed ferret (Mustela nigripes) (p. 455-464). In: Olney P.J.S., Mace G.M. \& Feistner A.T.C. (Eds). Creative Conservation. Dordrecht: Springer. https://doi.org/10.1007/978-94-011-0721-1_27

Moberg G.P. \& Mench J.A. (2000) The biology of animal stress: basic principles and implications for animal welfare. Wallingford: CABI. $384 \mathrm{p}$.

Munksgaard L., Passile A.M., Rushen J., Thodberg K. \& Jensen M.B. (1997) Discrimination of people by dairy cows based on handling. Journal of Dairy Science, 80: 1106-1112. https://doi.org/0.3168/jds.S0022-0302(97)76036-3

Neto G.S.F., Barros A.C., Sobroza T.V., Neves P.U.C., Phillips M.J. \& Guimarães E.F. (2020) Period of the day and food-based enrichment affect behaviour activity of Tamandua tetradactyla in captivity? Pesquisa e Ensino em Ciências Exatas e da Natureza, 4: e1498.

http://dx.doi.org/10.29215/pecen.v4i0.1498

Neto G.S.F., Oliveira R.C., Barros A.C., Ribeiro R.P., Melul R. \& Nomura F. (2021) Lutar, esconder ou correr: como Tamandua tetradactyla (Mammalia) em cativeiro responde a pistas indiretas de um predador? Pesquisa e Ensino em Ciências Exatas e da Natureza, 5: e1689. http://dx.doi.org/10.29215/pecen.v5i0.1689 
Ödberg F.O. (1984) Neurochemical correlates of stereotyped behaviour in voles. Applied Animal Behaviour Science, 13: 1-2.

Reboreda J.C. \& Fernandez G.J. (1997) Sexual, seasonal and group size differences in the 390 allocation of time between vigilance and feeding in the greater rhea (Rhea americana). Ethology, 103: 198-207.

Renison D., Valladares G. \& Martella M.B. (2010) The effect of passage through the gut of the greater rhea (Rhea americana) on germination of tree seeds: implications for forest restoration. Emu, 110: 125-131. http://dx.doi.org/10.1071/MU09090

Sekar M., Rajagopal T. \& Archunan G. (2008) Influence of zoo visitor presence on the behavior of captive Indian gaur (Bos gaurus gaurus) in a zoological park. Journal of Applied Animal Welfare Science 11(4): 352-357. http://dx.doi.org/10.1080/10888700802330093

Shepherdson D.J., Carlstead K., Mellen \& Seidensticker J. (1993) The influence of food presentation on the behavior of small cats in confined environments. Zoo Biology, 12: 203216. https://doi.org/10.1002/zoo.1430120206

Short J., Bradshaw S.D., Giles J., Prince R.I.T. \& Wilson G.R. (1992) Reintroduction of macropods (Marsupialia: Macropodoidea) in Australia - a review. Biological Conservation, 62: 189-204. https://doi.org/10.1016/0006-3207(92)91047-V

Webb J. (2020) Training Animals in Captivity or the Wild, so They Can Return to the Wild. Zoo Animal Learning and Training, 1-289: 308.

Wilcoxon F. (1992) Individual Comparisons by Ranking Methods. In: Kotz S. \& Johnson N.L. (Eds). Breakthroughs in Statistics. Springer Series in Statistics (Perspectives in Statistics). New York: Springer. https://doi.org/10.1007/978-1-4612-4380-9_16

Wolf C.M., Griffith B., Reed C. \& Temple S.A. (1996) Avian and mammalian translocations: update and reanalysis of 1987 survey data. Conservation Biology, 10: 1142-1154.

https://doi.org/10.1046/J.1523-1739.1996.10041142.X 